\title{
Plasma oxytocin levels and anxiety in patients with major depression
}

\author{
G. Scantamburlo ${ }^{a, *}$, M. Hansenne ${ }^{b}$, S. Fuchs ${ }^{a}$, W. Pitchot $^{a}$, P. Maréchal ${ }^{a}$, \\ C. Pequeux ${ }^{c}$, M. Ansseau ${ }^{a}$, J.J. Legros ${ }^{c}$
}

${ }^{a}$ Department of Psychiatry, University of Liège, Psychoneuroendocrinology Unit, CHU of Liège, B35, B-4000 Liège, Belgium ${ }^{\mathrm{b}}$ Department of Cognitive Sciences, Faculty of Psychology, University of Liège, B-4000 Liège, Belgium

'Department of Endocrinology, University of Liège, Psychoneuroendocrinology Unit, CHU of Liège, B35, B-4000 Liège, Belgium

Received 11 August 2006; received in revised form 25 January 2007; accepted 27 January 2007

\author{
KEYWORDS \\ Oxytocin; \\ Anxiety; \\ Major depression; \\ Neurohypophysis
}

\begin{abstract}
Cerebrospinal fluid and plasmatic levels of oxytocin (OT) have been found to change in mood disorders. In post-mortem studies, the numbers of OT-expressing neurons in the paraventricular nucleus have been reported to be increased. Moreover, OT is considered as an endogenous antistress hormone. It has also revealed antidepressive effects. OT may contribute to the dysregulation of the HPA system in major depression. The aim of the study was to assess a possible relationship between anxiety and plasma oxytocin (OT) levels in depressive patients. Severity of depression was estimated with the Hamilton Depression Rating Scale and anxiety by using the Spielberger State-Anxiety Inventory. Results showed a significant negative correlation between oxytocin and the scored symptoms depression $(r=-0.58, p=0.003)$ and anxiety $(r=-0.61, p=0.005)$.

(c) 2007 Elsevier Ltd. All rights reserved.
\end{abstract}

\section{Introduction}

Oxytocin (OT) is a nonapeptide synthetised in the magnocellular and the parvocellular neurons of the paraventricular and supraoptic nuclei of the hypothalamus and released directly into bloodstream from axon terminals in the posterior

*Corresponding author. Tel.: +3243667960; fax: +3233667283.

E-mail address: gabrielle.scantamburlo@ulg.ac.be

(G. Scantamburlo). pituitary. Hormones classically confined to the magnocellular-neurohypophysial system are found in the parvocellularlong portal system and are known to be implicated in the hypophysiotropic control of ACTH release. Apart from its presence in the hypothalamus, OT is found in several other brain regions (e.g., bed nucleus of the striae terminalis, central and medial nuclei of the amygdala, septum, hippocampus), suggesting that it has a role in neurotransmission, psychogenic stress and anxiety (Amico et al., 2004).

In animals, the neuropeptide oxytocin has been shown to exert behavioral and physiologic stress-attenuating and 
anxiolytic effects and, in addition, might prove to promote positive social interaction (Heinrichs et al., 2003). Research in humans remains relatively limited; however, initial studies suggest similar stress-reducing effects of the neuropeptide in humans (Uvnas-Moberg, 1997; Legros, 2002; Heinrichs et al., 2003), so that it is considered as an endogenous antistress neuropeptide in humans (Legros, 2001) and emerges in the pattern of the stress response.

Some studies have also revealed its antidepressive (Arletti and Bertolini, 1987) and antistress effects. There is speculation that some symptoms commonly reported in depression (social withdrawal, reduced appetite, cognitive impairment) may reflect central OT function (Bell et al., 2006). There are relatively few data on plasma OT levels in depression. Zetzsche et al. (1996) have shown a significant reduction of plasma OT in 12 depressed patients compared with age-matched controls. In addition, a blunting of OT secretion may be functionally related to the loss of appetite and the reduction of libido in depressive illness. In contrast, no significant difference was found between mean plasma levels of OT in depressed patients $(N=52)$ and controls $(N=37)$ by van Londen et al. (1997), but Andeberg and Uvnas-Moberg (2000) reported a negative correlation between the levels of OT and the symptom scores of anxiety and depression in female fibromyalgia syndrome patients. In addition, a positive correlation has been found between the levels of OT and happiness. Concerning CSF concentrations of OT, no significant difference was found between depressed patients and normal controls (Purba et al., 1996). In postmortem studies, the numbers of AVP- and OT-expressing neurons in the paraventricular nucleus of the hypothalamus have been reported to be increased (Purba et al., 1996).

Previous researchers have found evidence that indicates a relationship between OT and mood disorders, but more direct examination is needed. The present study will investigate further the possible relationship using the data from a sample of 25 patients that we have reported on in the previous paper (Scantamburlo et al., 2005). The study supported partially the hypothesis of a reduced vasopressinergic activity in depression. Moreover, we did not find any influence of acute apomorphine or clonidine injections on AVP- or OT-neurophysins in the depressed patients compared with controls.

\section{Method}

\subsection{Subjects}

The study was performed in 25 major depressive patients, representing consecutive admissions to the Biological Psychiatry and Psychopharmacology Unit of the University of Liège, Belgium. The diagnosis of major depressive disorder was based on the DSM-IV criteria and the selfquestionnaire from the Mini International Neuropsychiatric Interview. Moreover, patients had a score of at least 17 on the 17-item Hamilton Depression Rating Scale (HDRS) (Hamilton, 1960). The sample included 18 women with a mean age of 41.4 years (S.D. $=11.3$ ) and 4 men with a mean age of 38.6 years (S.D. $=10.4)$, with ages ranging from 19 to 59 years. Among the 18 women, 12 were premenopausal and 6 were postmenopausal. All premenopausal women were investigated during the first phase of the menstrual cycle as assessed by the patient-reported date of last menstruation. Patients with irregular menses were excluded.

All patients were free of medical illness as evidence by history, medical examination, ECG, chest X-ray, EEG, and routine laboratory tests. They had also been free of drugs, including benzodiazepines, for at least 2 weeks at the time of the study. Patients with a basal blood pressure $<100 / 70 \mathrm{mmHg}$ were excluded from the study.

The protocol was approved by the Ethical Committee of the University of Liège, Belgium. All patients were fully informed of the purpose of the study and gave written consent.

\subsection{Neuroendocrine procedure}

The patients arrived at the Biological Psychiatry and Psychopharmacology Unit half an hour before the blood sample were drawn, which was done at $0800 \mathrm{~h}$ in the morning. Blood samples were centrifuged within $2 \mathrm{~h}$ and the serum was immediately frozen and kept at $-18^{\circ} \mathrm{C}$ until analysis. OT was measured as previously described with a double antibody radioimmunoassay. Intraassay variability was $3.41 \%$ and interassay variability was $2.84 \%$. The sensitivity of OT enzyme-immunoassay is $1 \mathrm{pmol} / \mathrm{l}$.

\subsection{Anxiety assessment}

Self-report anxiety was determined by the State-Trait Anxiety Inventory (STAI) (Spielberger et al., 1983). The STAI offers a reliable and widely used measure of anxiety. It is a well-standardized, 40 item, self-report instrument designed to measure both state and trait anxiety. For the State Anxiety Inventory (STAI-A), subjects are asked how they feel at the time being questioned.

\subsection{Statistical analyses}

The relationship between OT values and the psychological data were assessed by Pearson product-moment correlation coefficients (two-tailed) and performed using Statistica for Windows (Statsoft Inc 1993, Stasoft, Tulsa, USA).

\section{Results}

The mean OT plasma concentrations were $3.67 \pm 1.34 \mathrm{ng} / \mathrm{ml}$.

The mean score of HDRS was 30.9 (S.D. = 4.8). The correlation between OT and HDRS among 25 patients appears in Fig. 1. Results showed a significant negative correlation between the symptom depression and plasma OT levels $(r=-0.58, p=0.003)$.

The mean score of STAI-A was 57.68 (S.D. $=12.17$ ). The correlation between OT and STAI-A among 25 patients appears in Fig. 2. Results showed a significant negative correlation between anxiety and OT levels $(r=-0.61$, $p=0.005)$. 


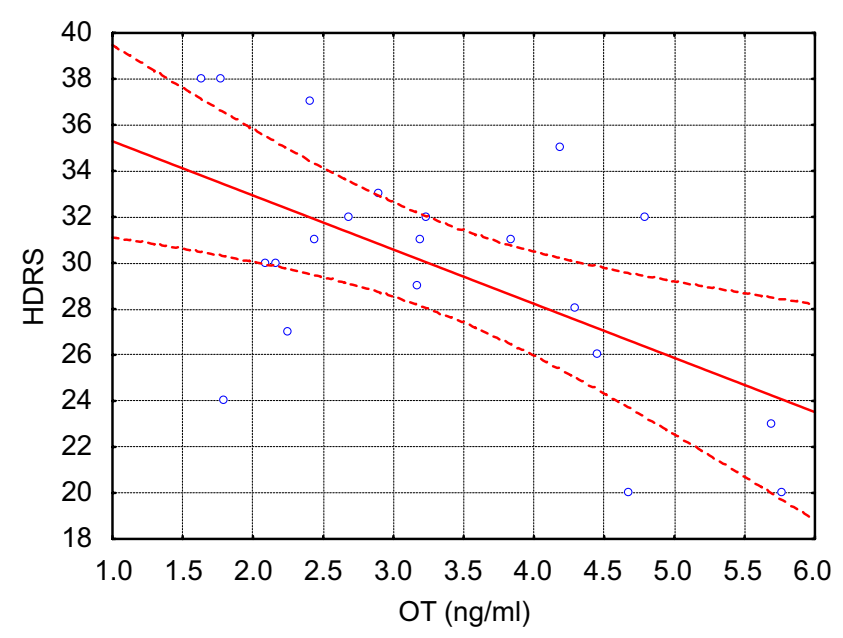

Fig. 1 Correlation between OT and HDRS among 25 patients $(r=-0.58, p=0.003)$.

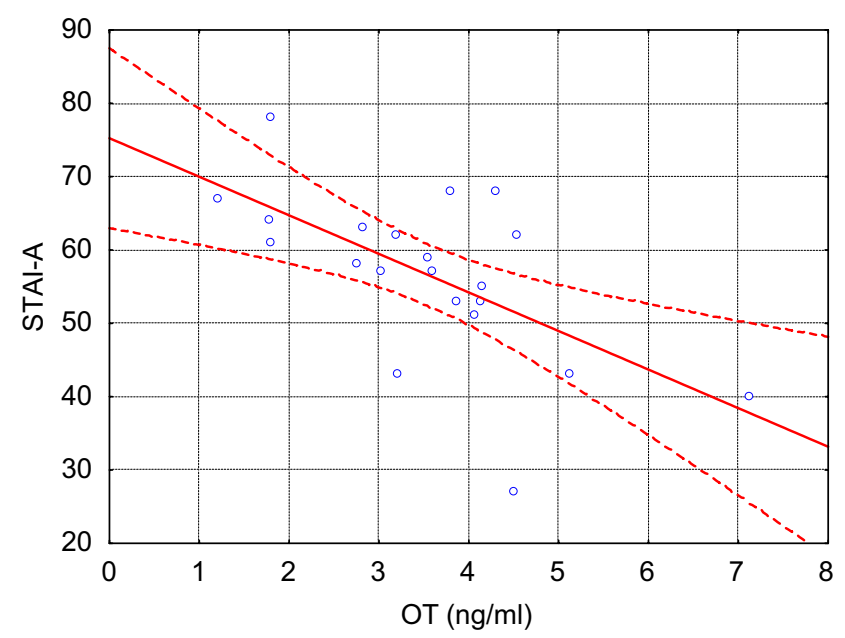

Fig. 2 Correlation between OT and STAI-A among 25 patients $(r=-0.61, p=0.005)$.

\section{Discussion}

The mean finding of the present study is a negative association between plasma OT levels in depressed patients and the scores of depression and anxiety. This is in accordance with our previous work and abnormalities of neurohypophysial secretions in major depressive disorder.

In animals, most studies suggest that OT attenuates glucocorticoid secretion (Windle et al., 1997) and diminishes anxiety (Neumann, 2002). In humans, OT has been proposed to play a role in the reduction of anxiety in anxious mothers during breast feeding (Altemus, 1995). Moreover, OT seems to enhance the buffering effect of social support on stress responsiveness (Heinrichs et al., 2003).

OT is involved in mood disorders and may also act as a potent secretagogue of ACTH release at the anterior lobe of the pituitary (Andeberg and Uvnas-Moberg, 2000). Intracerebral OT inhibits the stress-induced activity of the hypothalamic-pituitary adrenal (HPA) axis responsiveness suggesting an inhibitory influence of OT on stress-responsive neurohormonal system (Heinrichs et al., 2003). As OT is known to attenuate the stress response in humans, they postulated that the OT reduction in the depressed patients may contribute to the dysregulation of HPA system consistently found in major depression (Zetzsche et al., 1996).

While vasopressin is known as an ACTH stimulating factor synergistic to CRF, it is likely that endogenous oxytoninergic system, which can be activated by physiological and/or pharmacological manipulation, can "buffer" the stress activated vasopressin-ACTH-cortisol action (Legros, 2001; Heinrichs et al., 2003). A variety of neurotransmitters and neuropeptides influence the HPA axis. Central OT pathways are believed to facilitate anxiolysis and to attenuate the response of the HPA axis to psychogenic stressors. The medial amygdalia may be an important region for OT actions that govern a variety of social and stress behaviours (Amico et al., 2004). In major depression there is an upregulation in the HPA axis, which in turn down-regulates the 5-HT1A receptors and the activity of serotonin. The increased number of oxytocin neurons may be a compensatory physiological mechanism to balance and increase the serotoninergic activity in order to accomplish better mood and well-being.

Some limitations of the study need to be discussed. This is a clinical population of mostly women with limited generalizability to other populations. Other information, such as childbirth and lactation history, which may influence the oxytoninergic system, was not gathered or analyzed. The most important admission is that the study is correlational and that no direction of causality can be made.

Pharmacological or nonpharmacological activation of endogenous OT release might be considered as a way of alleviating stress (Legros, 2001, 2002). The OT and serotonin (5-hydroxytryptophan (5-HT)) systems are interconnected. Oxytocin fibers project to the raphe nuclei and OT may influence the secretion of 5-HT. Recent studies have demonstrated that OT is released in response to treatment with selective serotonin reuptake inhibitors (SSRI), opening up the possibility that OT also mediate further effects caused by serotonin (Uvnas-Moberg et al., 1999).

In conclusion, the present study supports the importance of OT in mechanism underlying anxiolysis. Future work focusing on male/female differences or looking at childbirth and lactation history could be done. A further study should explore OT administration. Neurendocrine research is also needed to elucidate the mode of action of OT, its antistress properties, and the interactions with the HPA axis and the monaminergic system. Finally, we should emphasize the effect size of the finding (Lawrence Erlbaum Associates, 1984). The magnitude of the r's suggests a strong association between OT and mood. These appear to be a robust finding that merit further research and may yield important clinical applications.

\section{Role of the funding source}

This study was supported by Grant from the "Fonds d'Investissements pour la Recherche Scientifique" (CHU of Liège) in the analysis of data.

\section{Conflict of interest}

All other authors declare that they have no conflicts of interest. 


\section{Acknowledgements}

The help of Mrs M.T. Hagelstein for her expert technical assistance is acknowledged. Special thanks to C. Scantamburlo.

\section{References}

Altemus, M., 1995. Neuropeptides in anxiety disorders: effect of lactation. Ann. N.Y. Acad. Sci. 771, 697-707.

Amico, J.A., Mantella, R.C., Vollmer, R.R., Li, X., 2004. Anxiety and stress response in female oxytocin deficient mice. J. Neuroendocrinol. 16, 319-327.

Andeberg, A.M., Uvnas-Moberg, K., 2000. Plasma oxytocin levels in female fibromyalgia syndrome patients. Z. Rheumatol. 59, 373-379.

Arletti, R., Bertolini, A., 1987. Oxytocin acts as an anti-depressant in two animal models of depression. Life Sci. 41, 1725-1730.

Bell, C.J., Nicholson, H., Mulder, R.T., Lutsy, S.E., Joyce, P.R., 2006. Plasma oxytocin levels in depression and their correlation with the temperament dimension of reward dependence. J. Psychopharmacol. 20, 600-656.

Hamilton, M., 1960. A rating scale for depression. J. Neurol. Neurosurg. Psychiatry 12, 56-62.

Heinrichs, M., Baumgartner, T., Kirschbaum, C., Ehlert, U., 2003. Social support and oxytocin interact to suppress cortisol and subjective responses to psychological stress. Biol. Psychiatry 54 , 1389-1398.

Lawrence Erlbaum Associates, 1984. Applied Multiple Regression/ Correlation Analysis for the Behavioral Sciences, second ed. (Hardcover).

Legros, J.J., 2001. Inhibitory effect of oxytocin on corticotrope function in humans: are vasopressin and oxytocin ying-yang neurohormones? Psychoneuroendocrinology 26, 649-655.
Legros, J.J., 2002. L'ocytocine: un moyen naturel de lutter contre le stress psychologique. Bull. Mem. Acad. R. Med. Belg. 157, 383-390.

van Londen, L., Goedkoop, J., van Kempen, G., FrankhuijzenSierevogel, A., Wiegant, V., van der Velde, E., 1997. Plasma levels of arginine vasopressin elevated in patients with major depression. Neuropsychopharmacology 17, 284-292.

Neumann, I.D., 2002. Involvement of the brain oxytocin system in stress coping: interactions with the hypothalamio-pituitaryadrenal axis. Prog. Brain Res. 139, 147-162.

Purba, J.S., Hoogendijk, W.J., Hofman, M.A., Swaab, D.F., 1996. Increased number of vasopressin- and oxytocin-expressing neurons in the paraventricular nucleus of the hypothalamus in depression. Arch. Gen. Psychiatry 53, 137-143.

Scantamburlo, G., Hansenne, M., Fuchs, S., Pitchot, W., Pinto, E., Reggers, J., Ansseau, M., Legros, J.J., 2005. AVP- and OTneurophysins response to apomorphine and clonidine in major depression. Psychoneuroendocrinology 30, 839-845.

Spielberger, C.D., Gorsuch, R.L., Lushene, R., Vagg, P.R., Jacobs, G.A., 1983. Manual for the State-Trait Anxiety Inventory. Consulting Psychologists' Press, Palo Alto, CA.

Uvnas-Moberg, K., 1997. Oxytocin linked antistress effectsthe relaxation and growth response. Acta Physiol. Scand. 161, $38-42$.

Uvnas-Moberg, K., Bjokstrand, E., Hillegaart, V., Ahlenius, S., 1999. Oxytocin as a possible mediator of SSRI-induced antidepressant effects. Psychopharmacology (Berl) 142, 95-101.

Windle, R.J., Shanks, N., Lightman, S.L., Ingram, C.D., 1997. Central oxytocin administration reduces stress-induced corticosterone release and anxiety. Endocrinology 138, 2829-2834.

Zetzsche, T., Frasch, A., Jirikowski, G., Murck, H., Steigner, A., 1996. Nocturnal oxytocin secretion is reduced in major depression. Biol. Psychiatry 39, 584. 